\title{
LA-UR-12-23946
}

Approved for public release; distribution is unlimited.

Title:

Author(s):
A New Gated X-Ray Detector for the Orion Laser Facility

\author{
Clark, David D. \\ Aragonez, Robert J. \\ Archuleta, Thomas N. \\ Fatherley, Valerie $\mathrm{E}$. \\ Hsu, Albert $\mathrm{H}$. \\ Jorgenson, H. J. \\ Mares, Danielle \\ Oertel, John A. \\ Oades, Kevin \\ Kemshall, Paul \\ Thomas, Philip \\ Young, Trevor \\ Pederson, Neal
}

Intended for:
SPIE Conference on Optics and Photonics, 2012-08-12/2012-08-16 (San Diego, California, United States)

Disclaimer:

Los Alamos National Laboratory, an affirmative action/equal opportunity employer,is operated by the Los Alamos National

Security, LLC for the National NuclearSecurity Administration of the U.S. Department of Energy under contract DE-AC52-06NA25396. By approving this article, the publisher recognizes that the U.S. Government retains nonexclusive, royalty-free license to publish or reproduce the published form of this contribution, or to allow others to do so, for U.S. Government purposes.

Los Alamos National Laboratory requests that the publisher identify this article as work performed under the auspices of the

U.S. Departmentof Energy. Los Alamos National Laboratory strongly supports academic freedom and a researcher's right to publish; as an institution, however, the Laboratory does not endorse the viewpoint of a publication or guarantee its technical correctness. 


\section{A New Gated X-Ray Detector for the Orion Laser Facility}

\section{SPIE Conference August 2012 San Diego CA}

David D. Clark, Robert Aragonez, Thomas Archuleta, Valerie Fatherley, Albert Hsu, Justin Jorgenson, Danielle Mares, John Oertel

Los Alamos National Laboratory, Los Alamos, NM Kevin Oades, Paul Kemshall, Philip Thomas, Trevor Young

Atomic Weapons Establishment, Aldermaston, UK

Neal Pederson

VI Control Systems,Los Alamos, NM 


\section{Imaging $\mathrm{X}$-rays is most easily done with small pinholes in a high-z substrate}

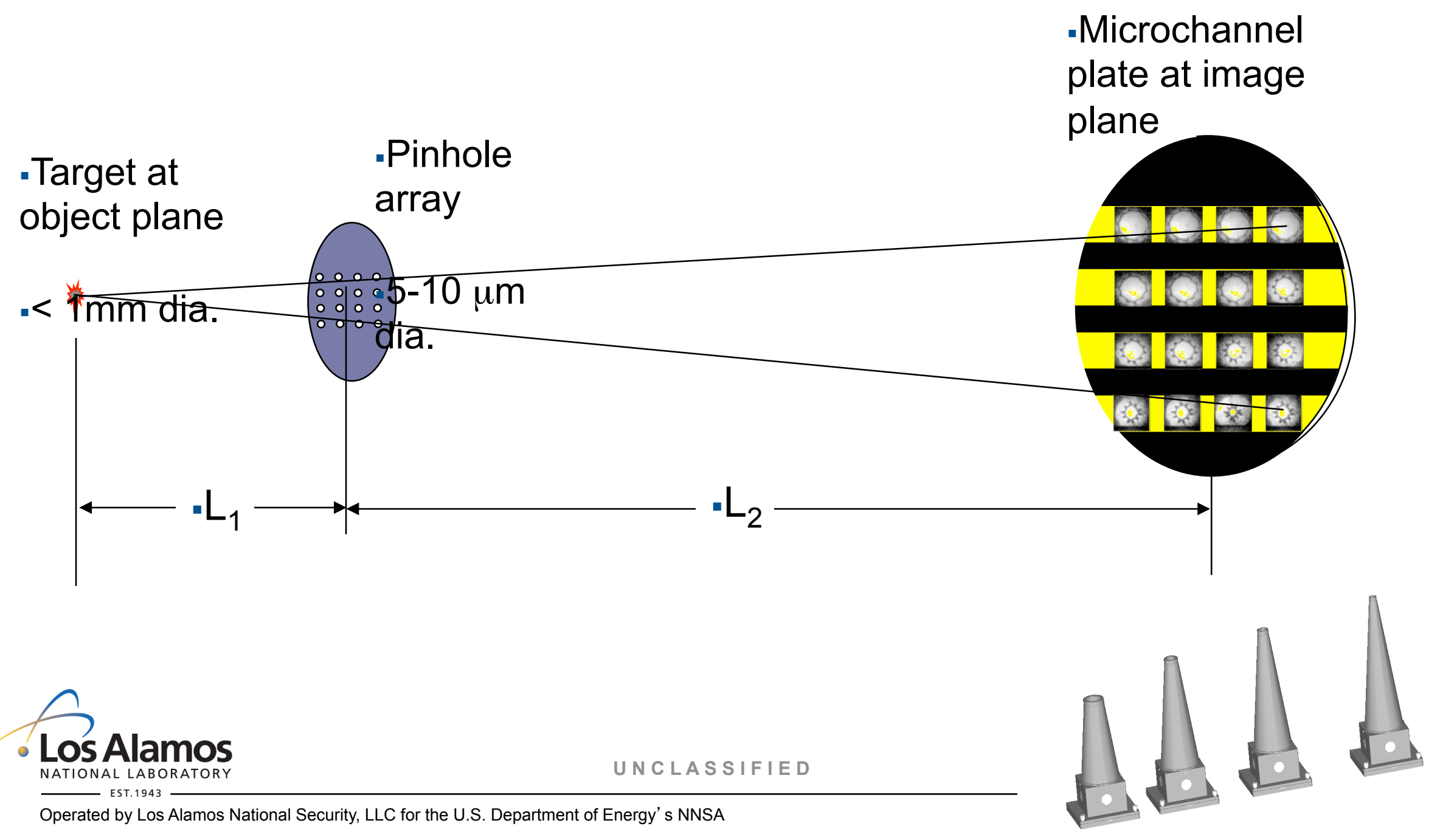




\section{Principle of Operation Front View}

-Gating is provided by launching a short (200 ps to several ns) 1kV voltage pulse across a microstrip transmission line coated on the MCP.

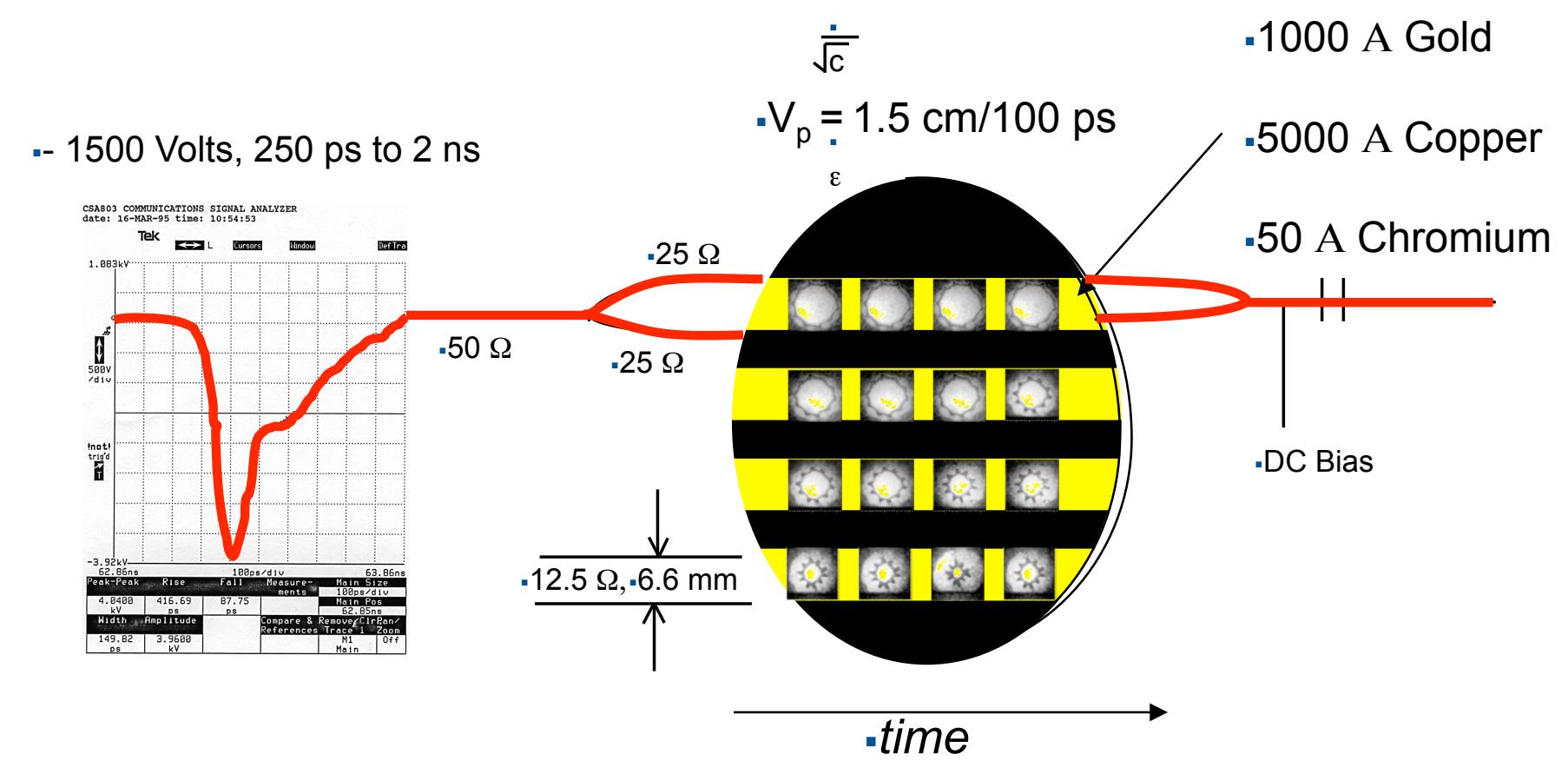




\section{The Orion Laser Facility}

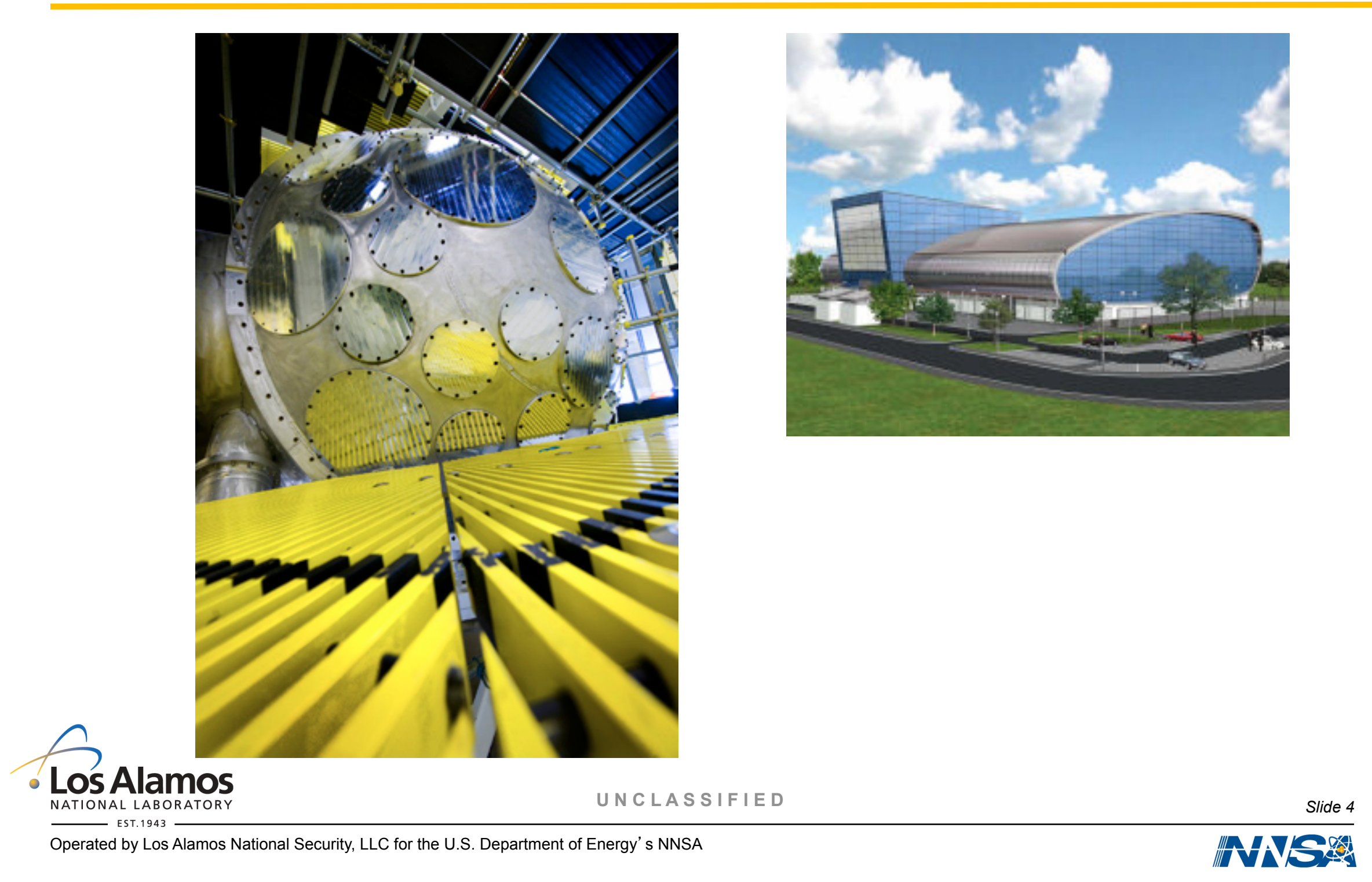




\section{The Orion Gated X-Ray Detectors}

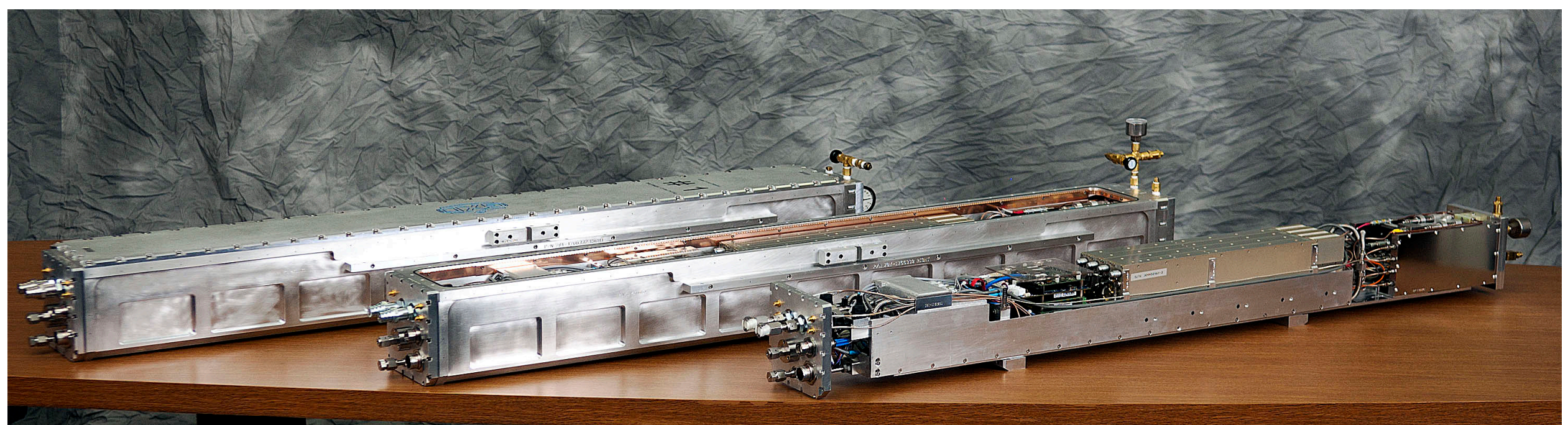

- LosAlamos 


\section{Orion GXD Block Diagram}

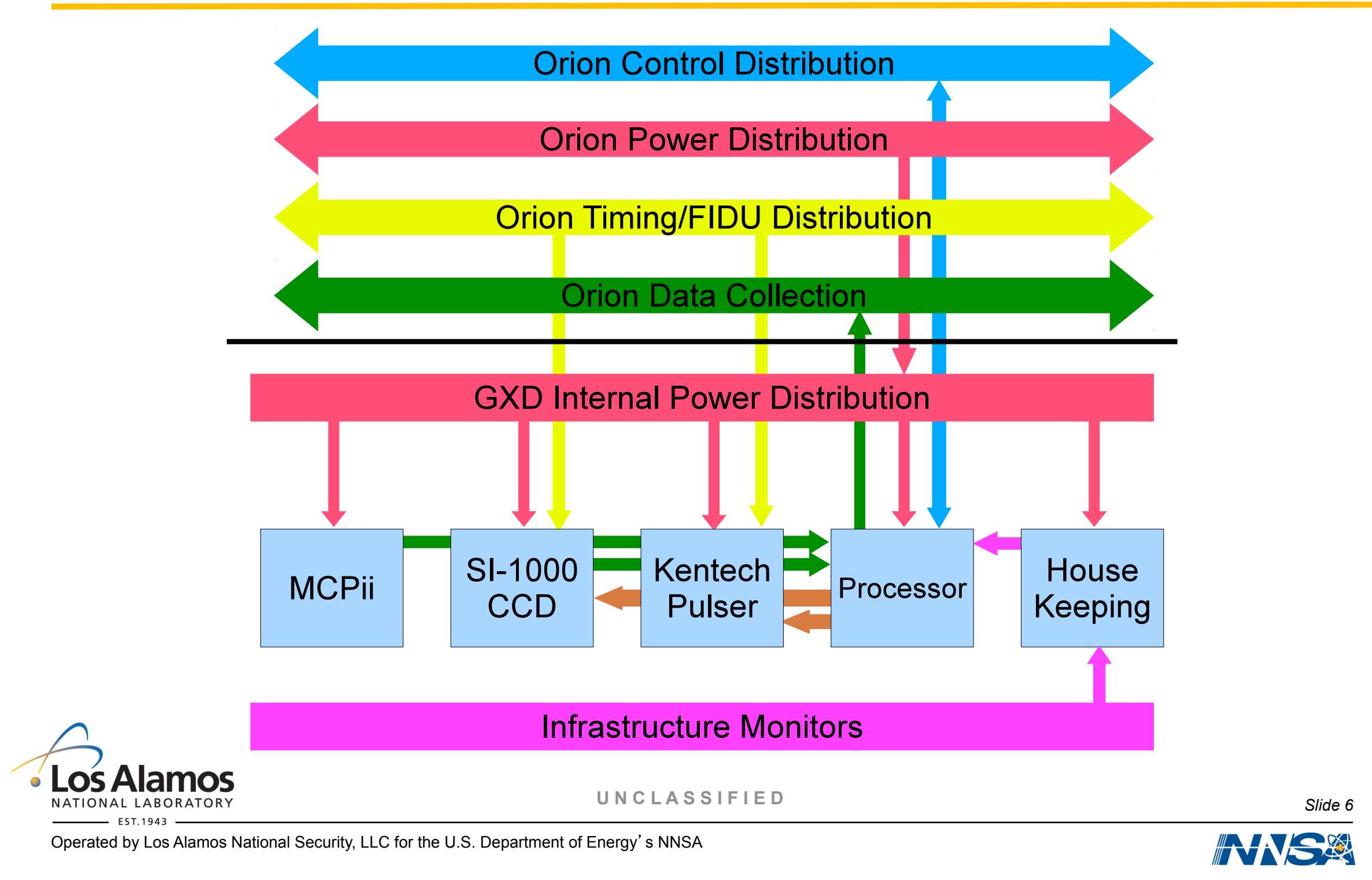




\section{Component Locations}
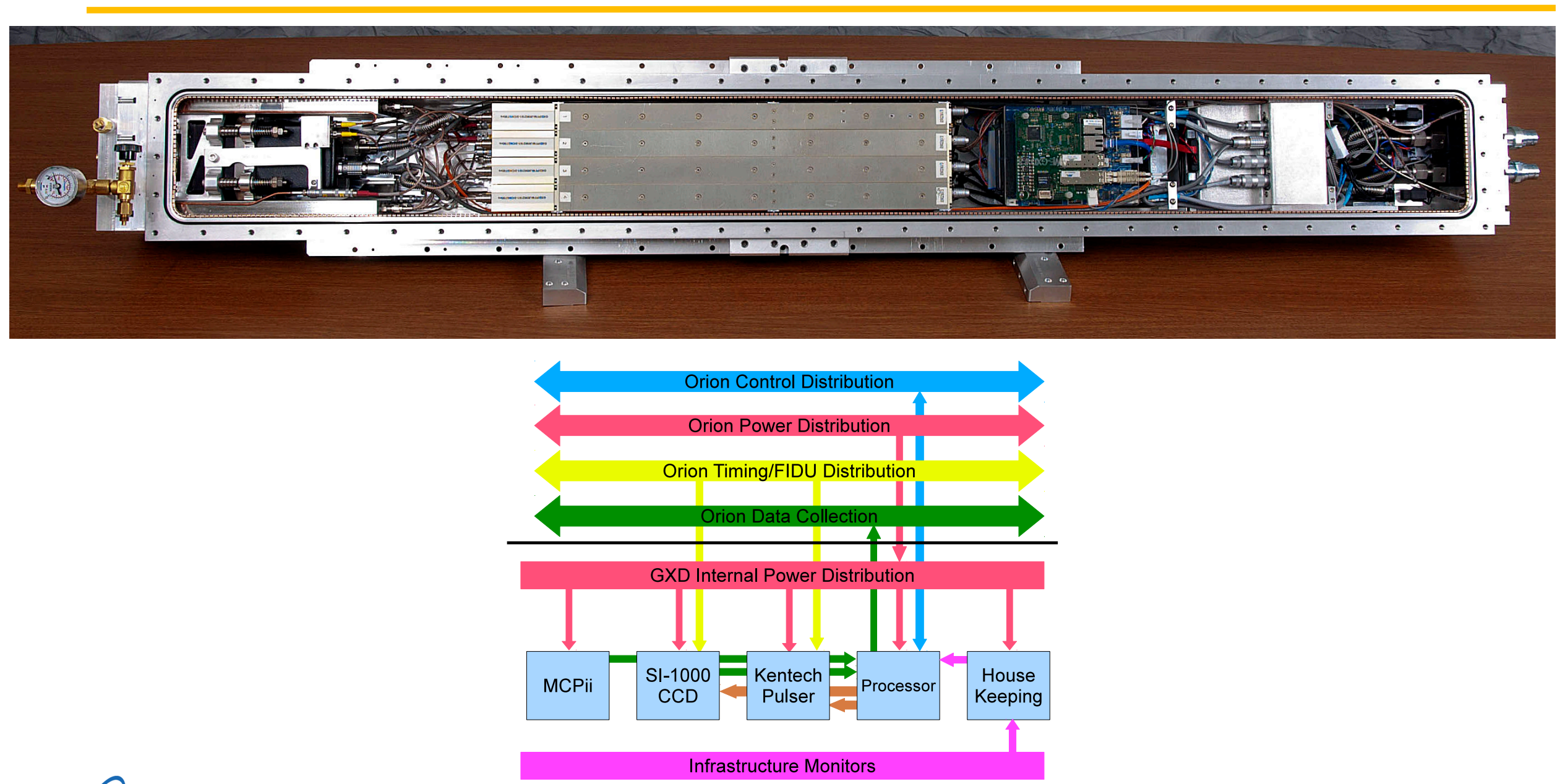

\section{- Los Alamos




\section{Head Improvements}

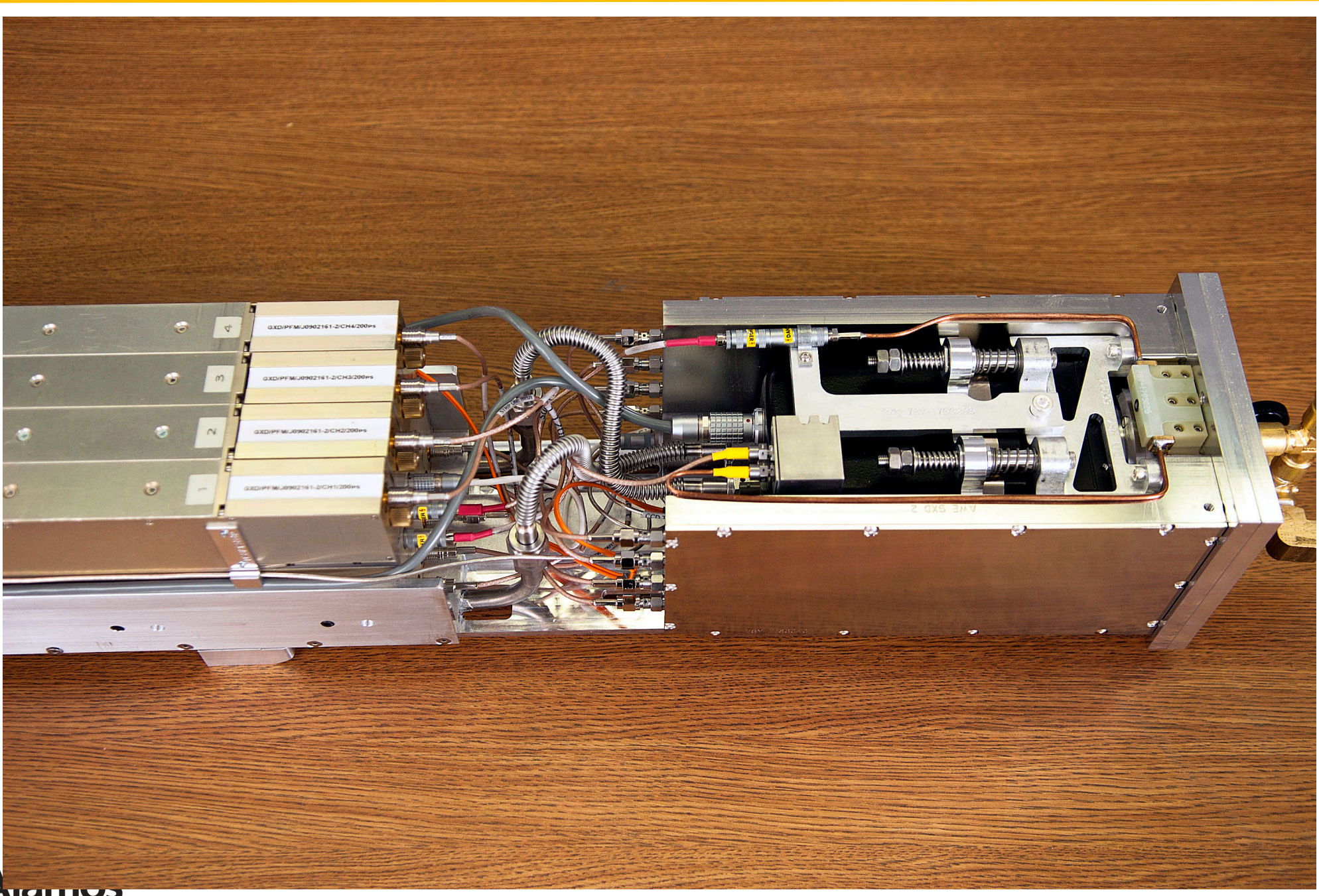




\section{Microwave Stripline Improvements}
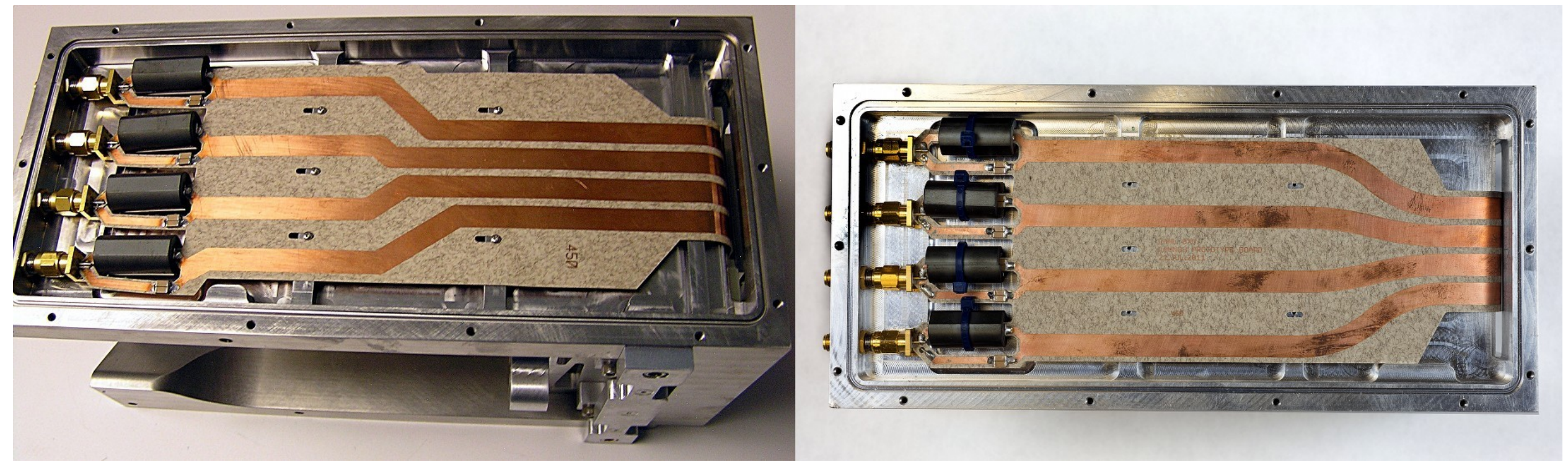

- Los Alamos 


\section{Connector Landing Design Improvements}
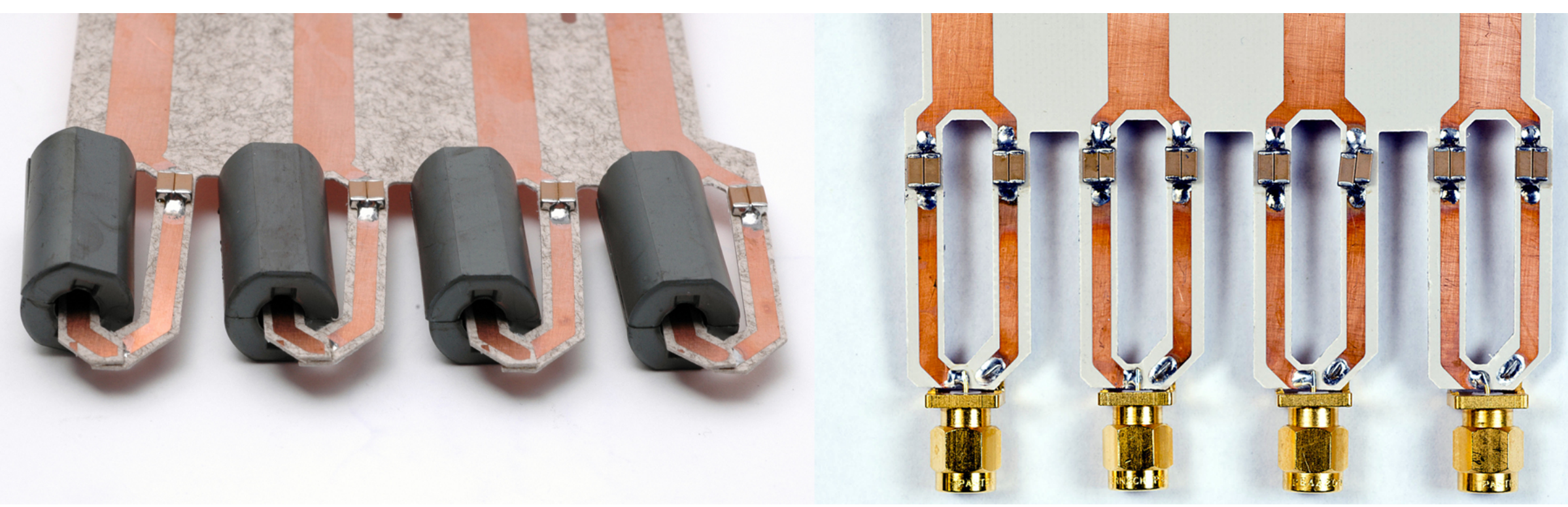

- LOSAlamos 


\section{Electrical Cross Talk Measurements}

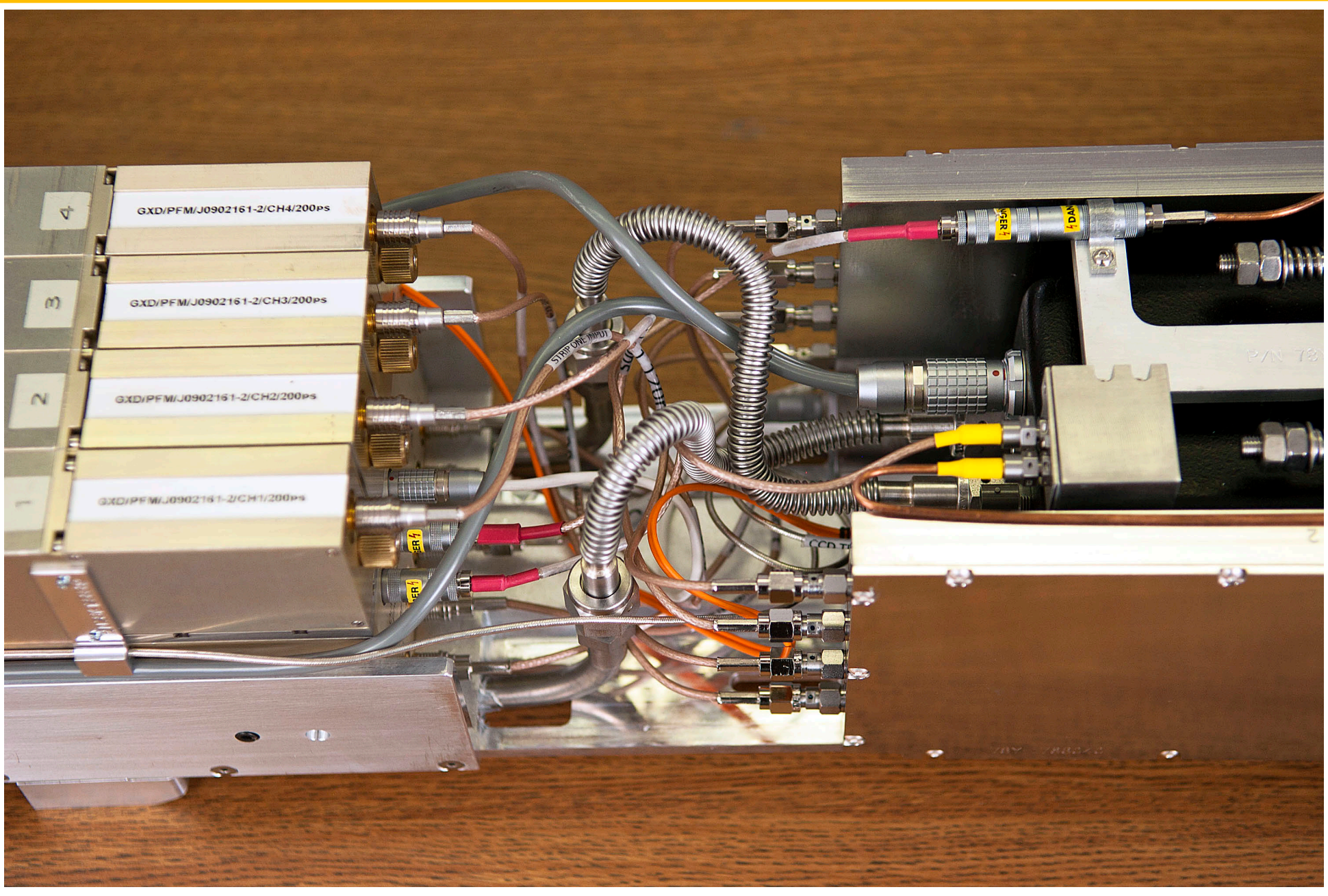




\section{Example Cross Talk Measurement Data}

Signal Coupled to Strip 2

Strip 1 Driven
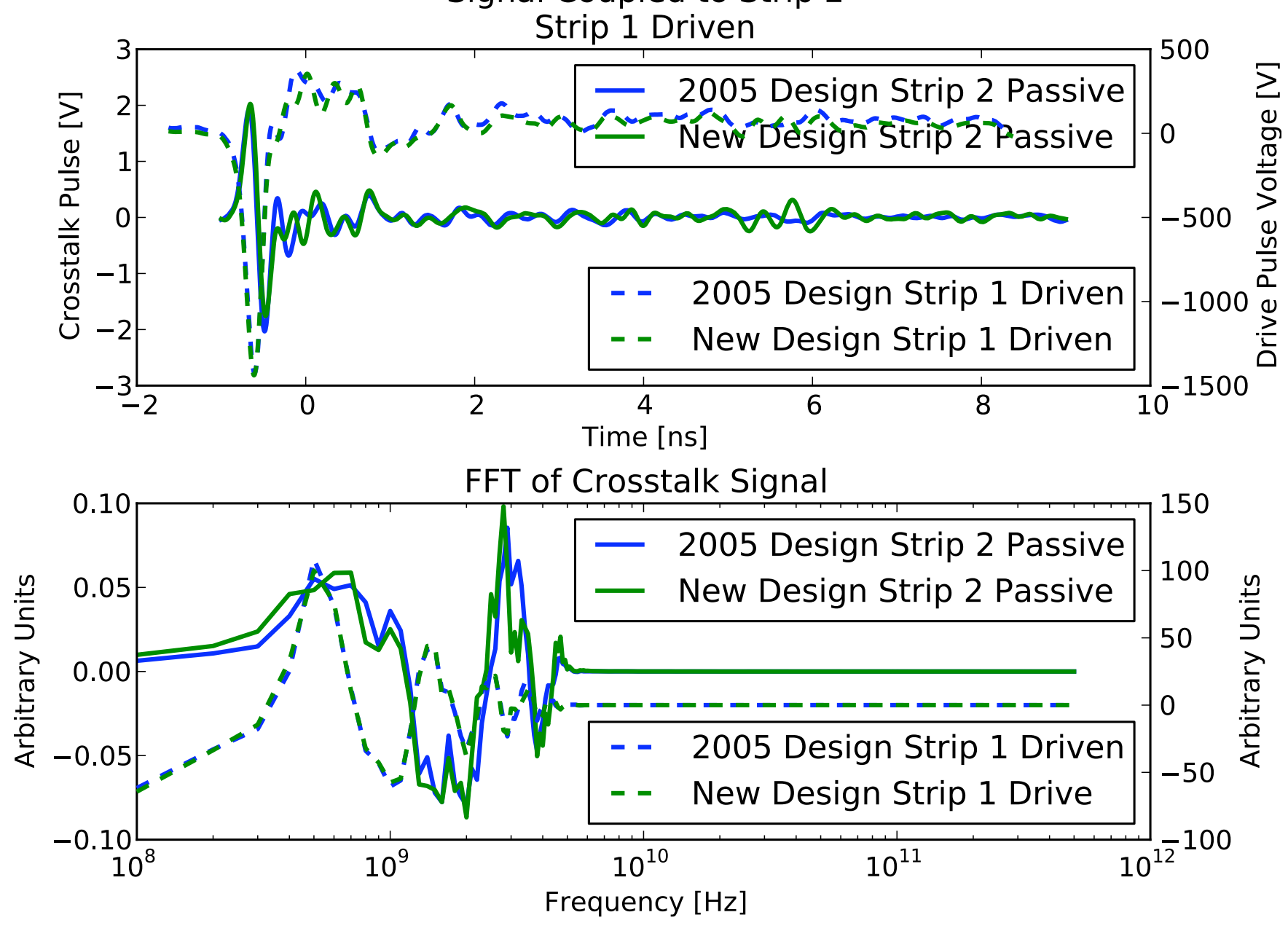


\section{Impedance Matching Measurement}

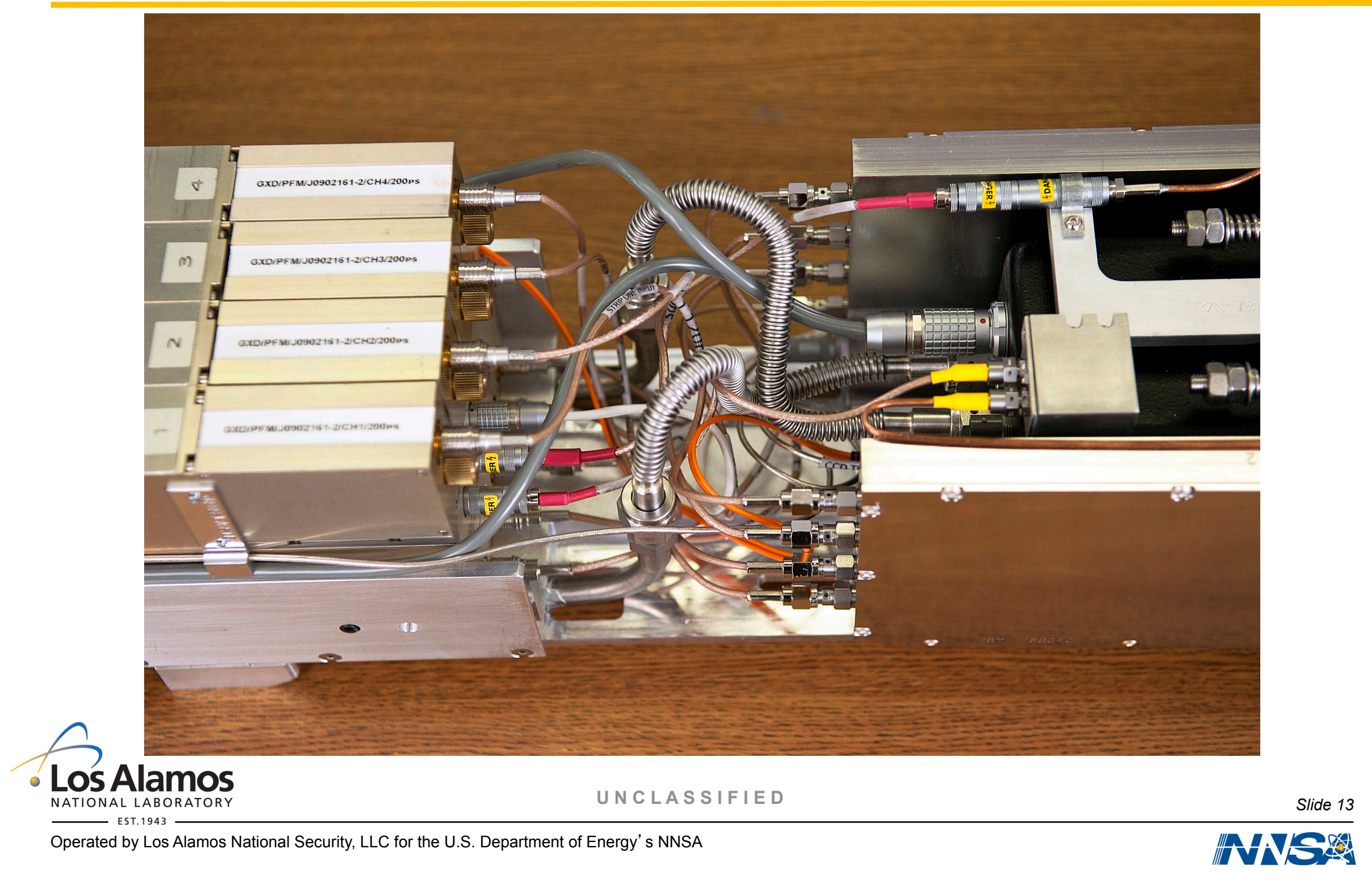




\section{Impedance Matching Data}

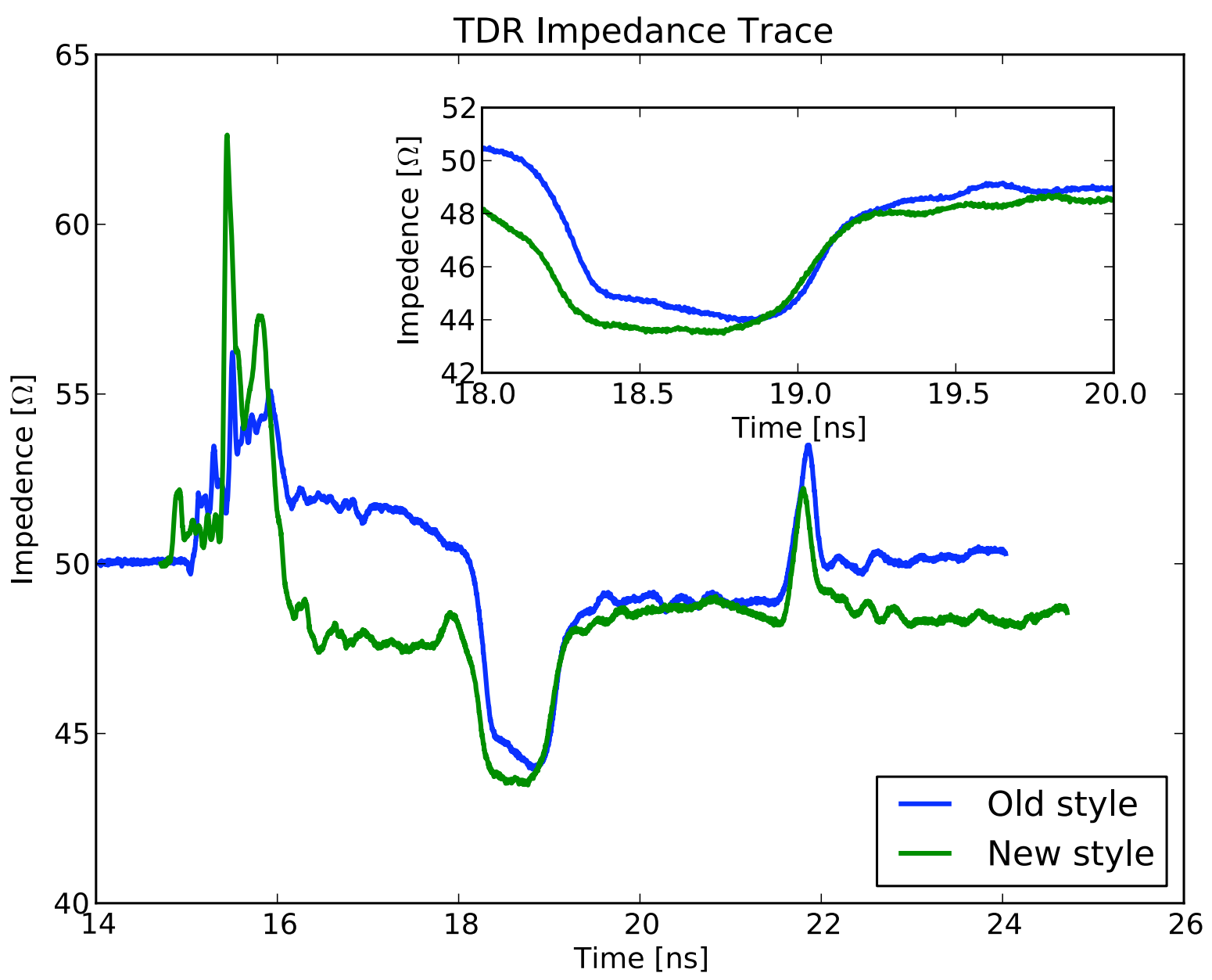




\section{Impedance Matching Data}

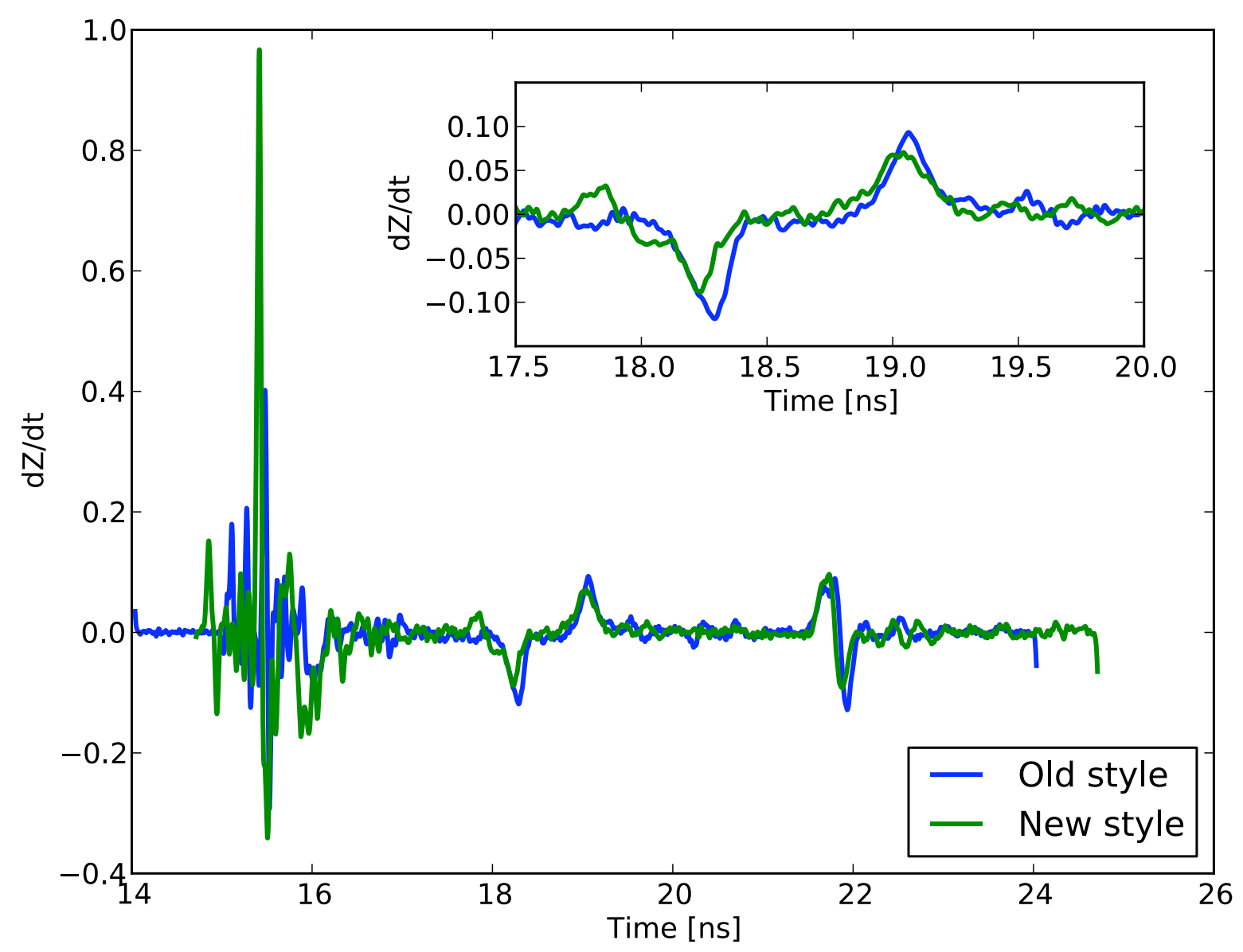




\section{Embedded Computer Improvements}

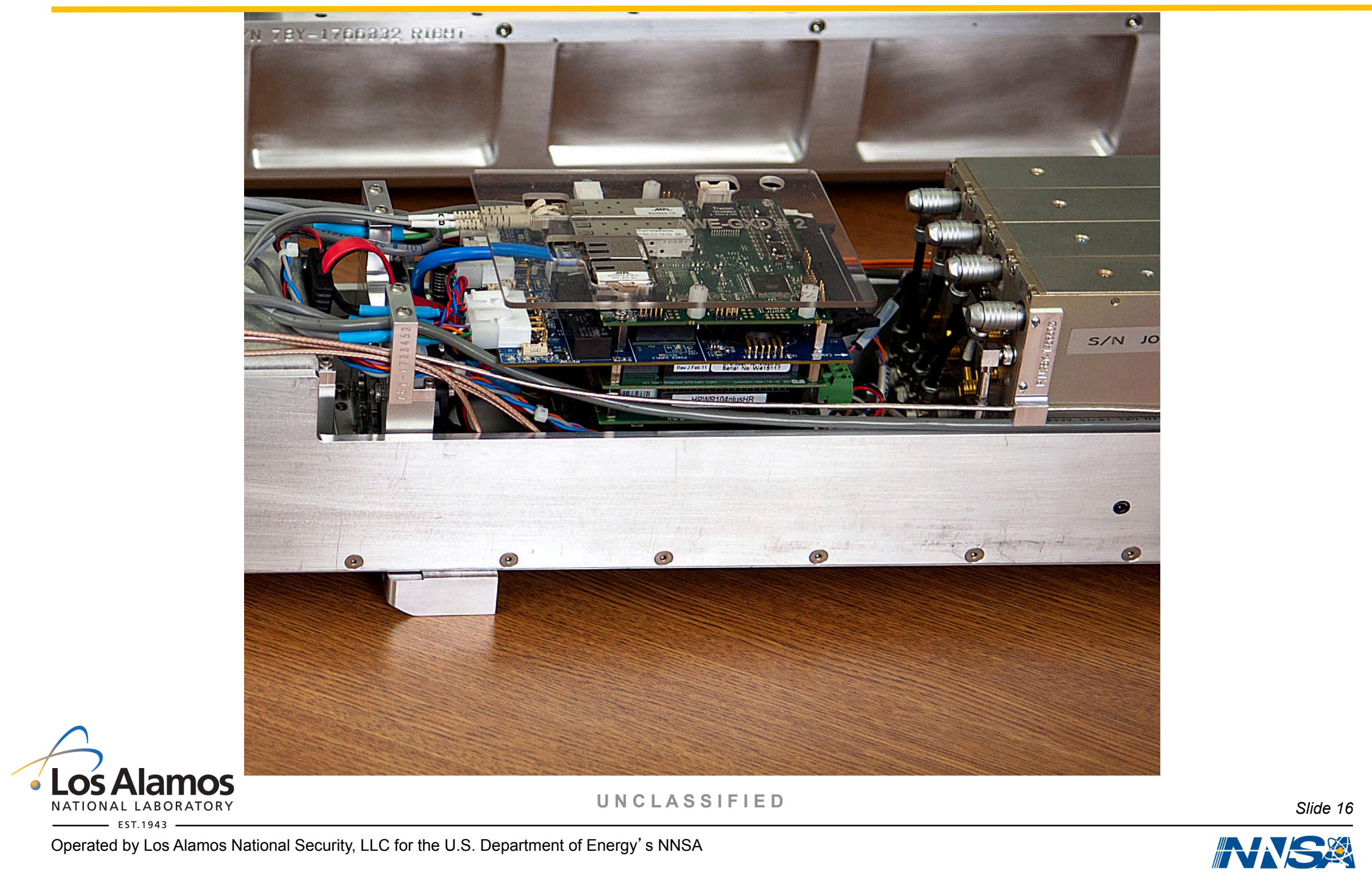




\section{PC/104+ Stack}
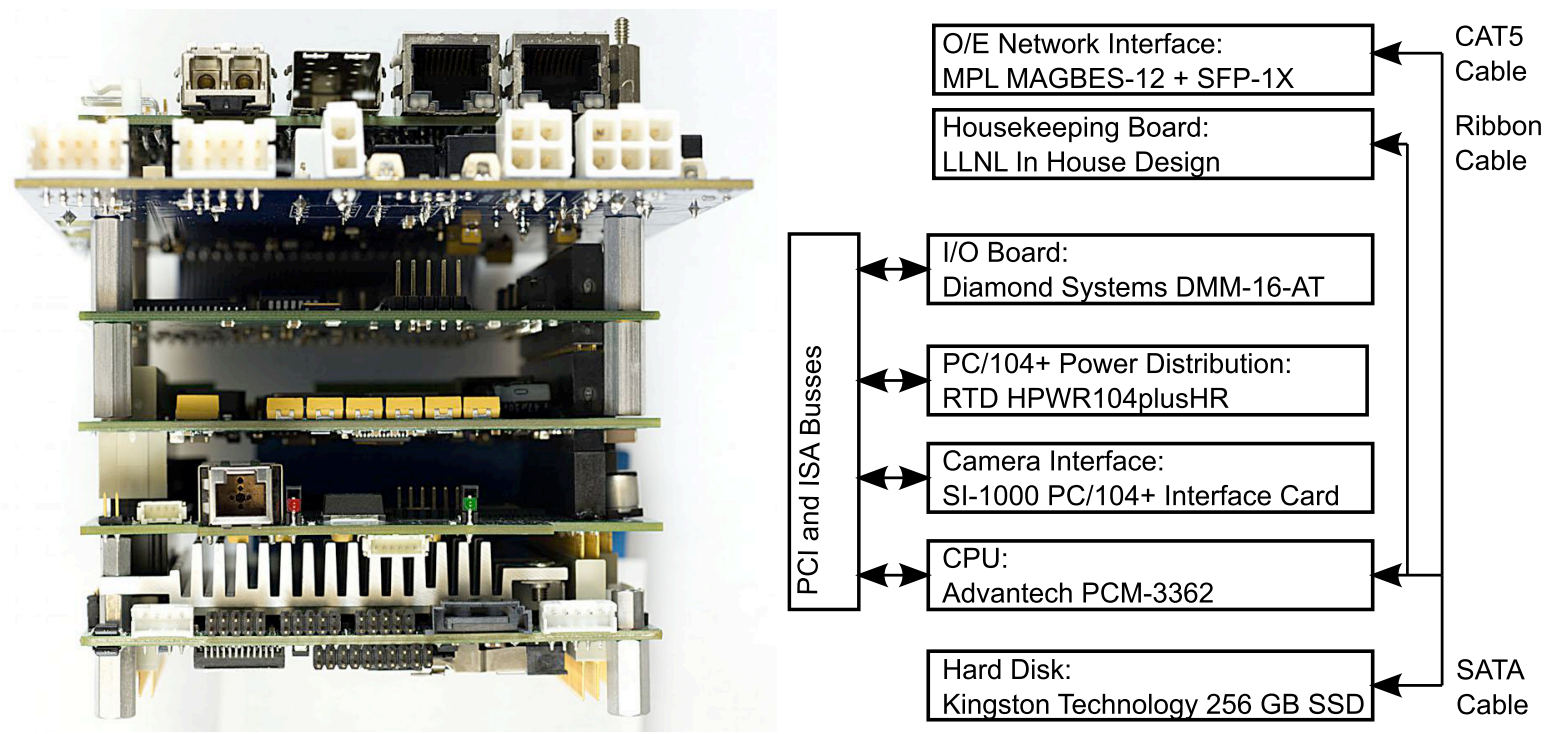


\section{Optical Network Switch}

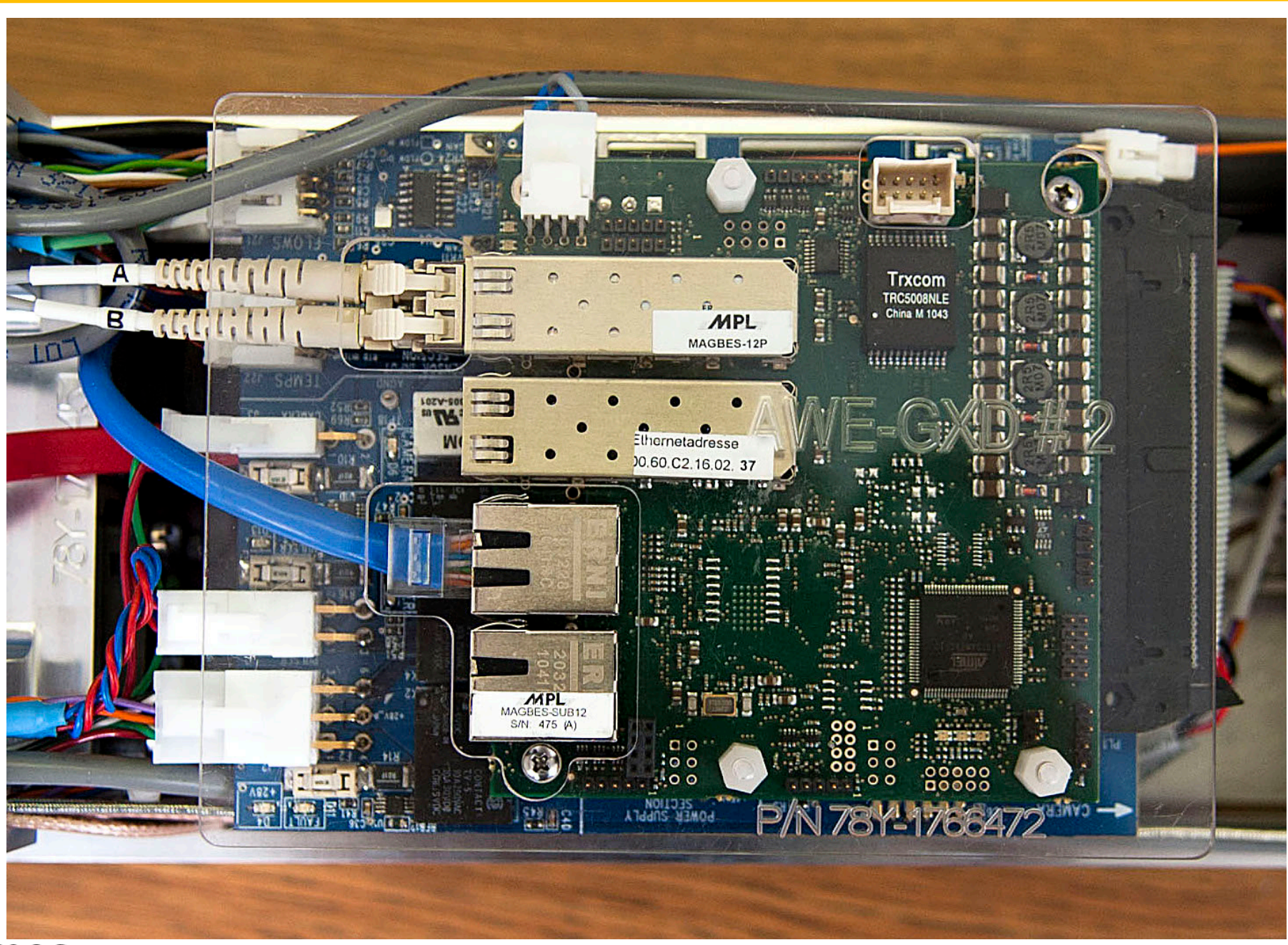




\section{Solid State Hard Disk}

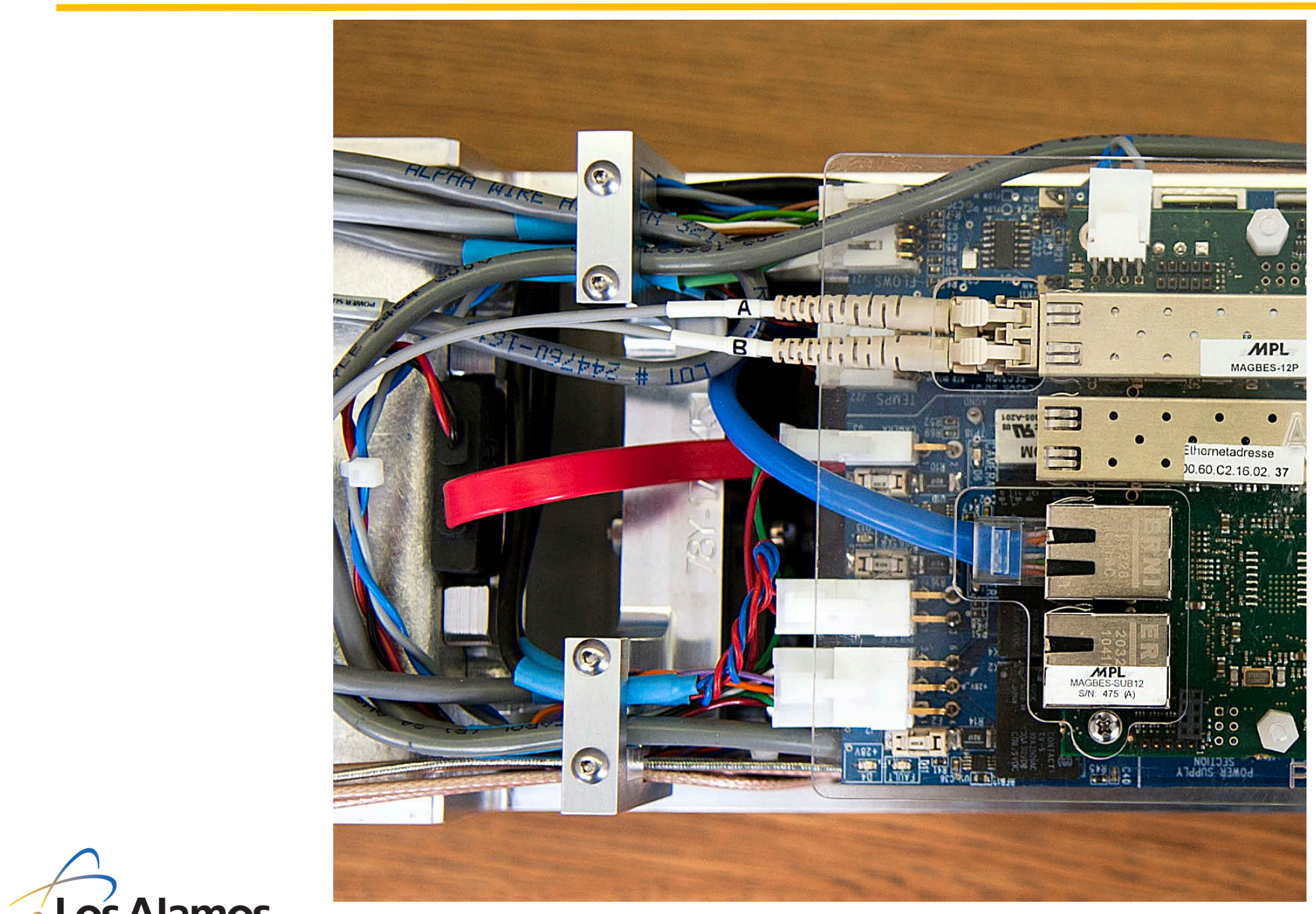




\section{Updated Control Software: LabView Interface}

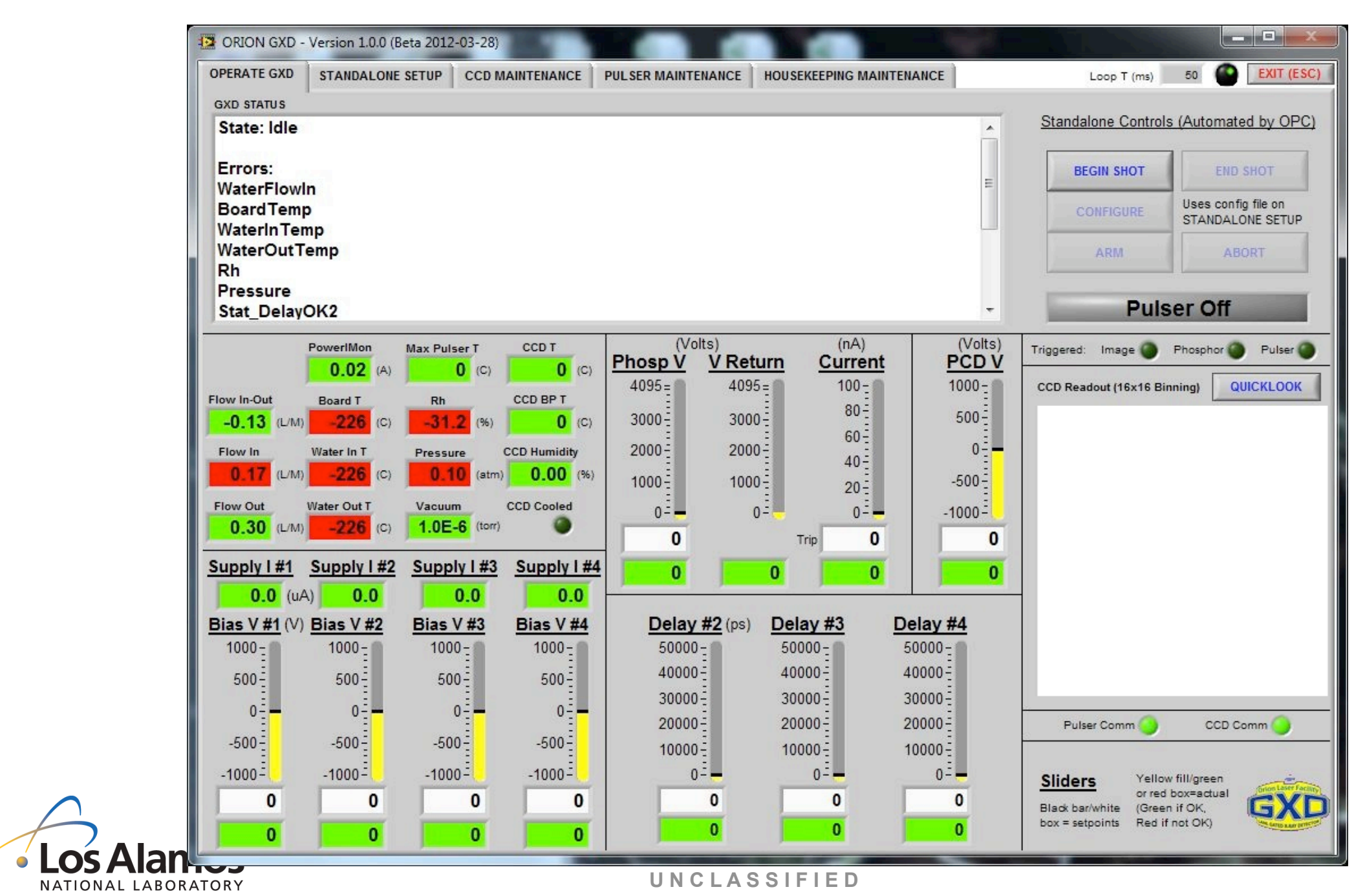




\section{Updated Software: OPC Interface}

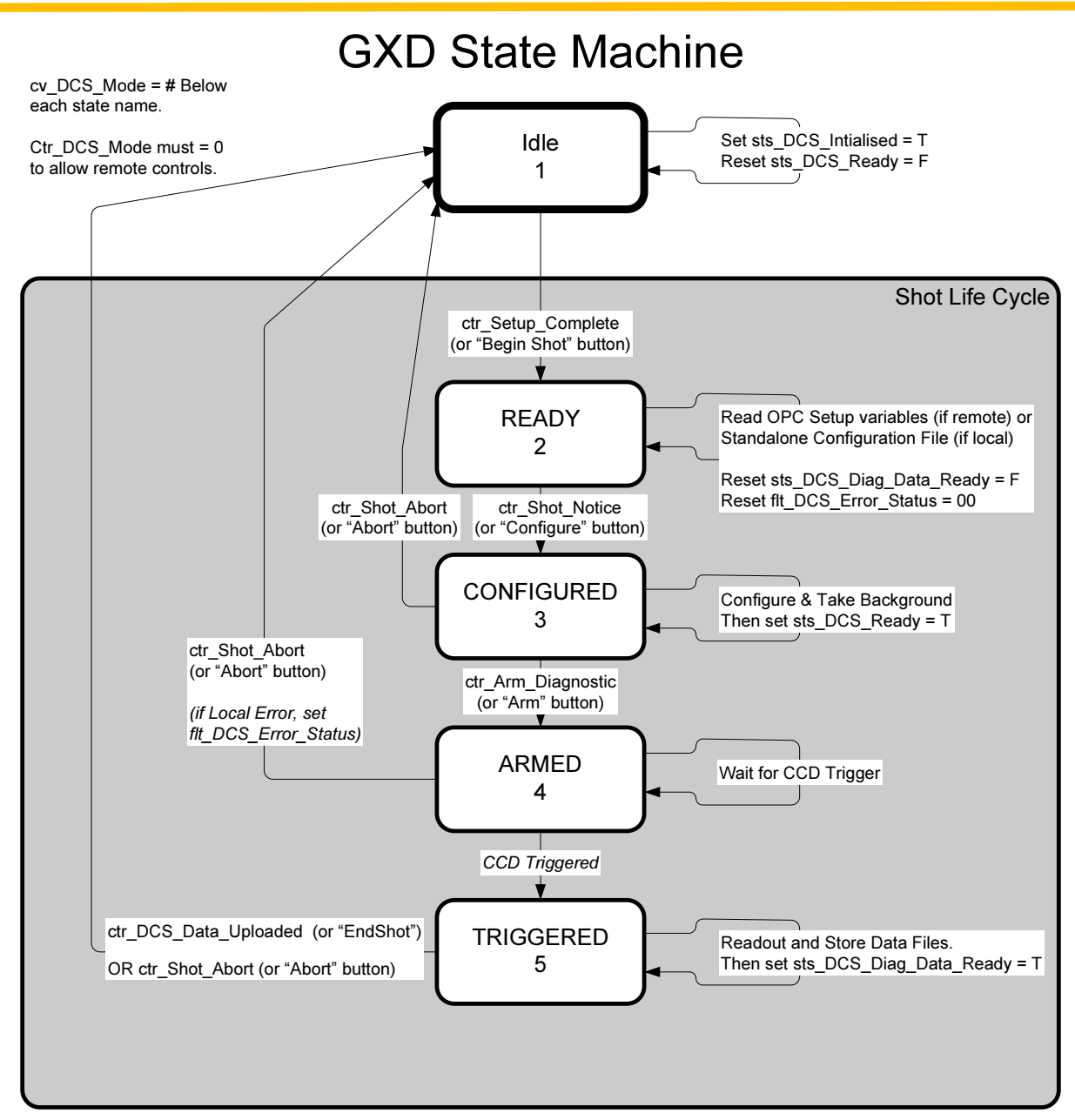




\section{Mechanical Improvements}




\section{When the Orion GXD was born...}

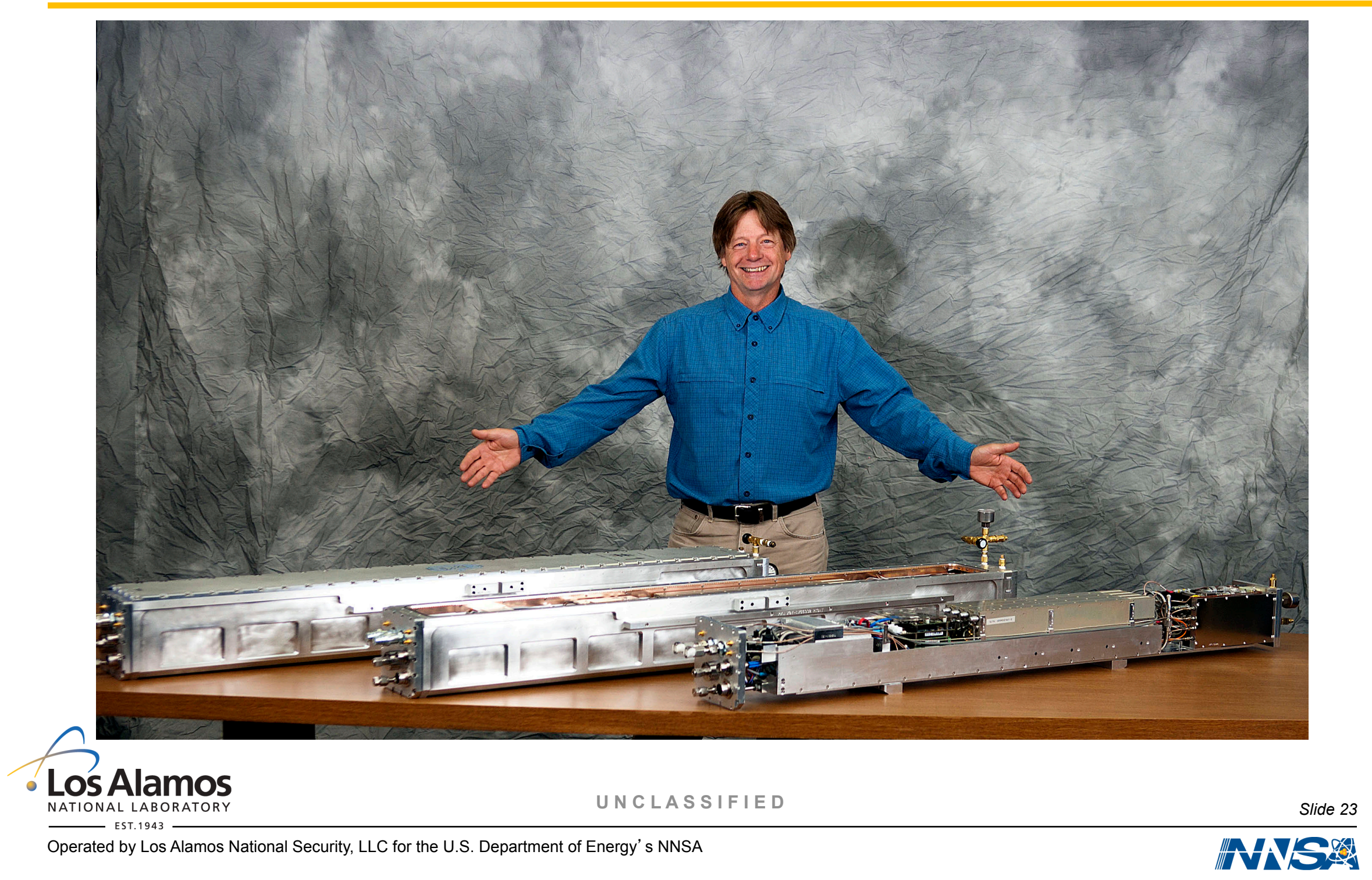




\section{Birth Stats...}

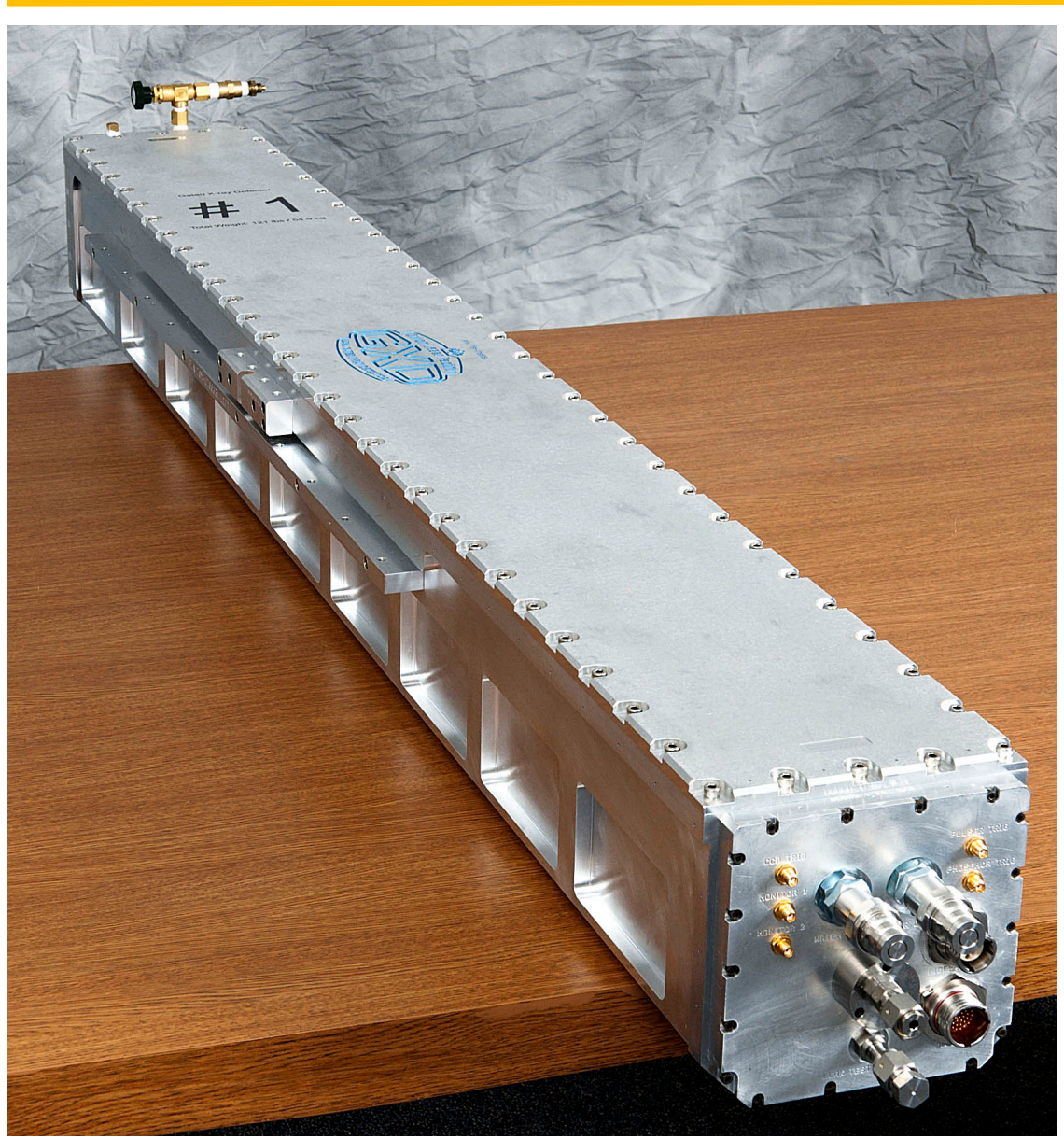

-Length: 60 inches $(152 \mathrm{~cm})$

-Lid: 12 pounds $(6 \mathrm{~kg})$

-Reduced 5 pounds $(2 \mathrm{~kg})$

-Air Box: 42 Pounds (19 kg)

-Reduced 13 pounds $(6 \mathrm{~kg})$

-Total Weight: 121 Pounds (63 kg)

-Reduced 18 pounds $(8 \mathrm{~kg})$ 


\section{Front and Rear Covers}

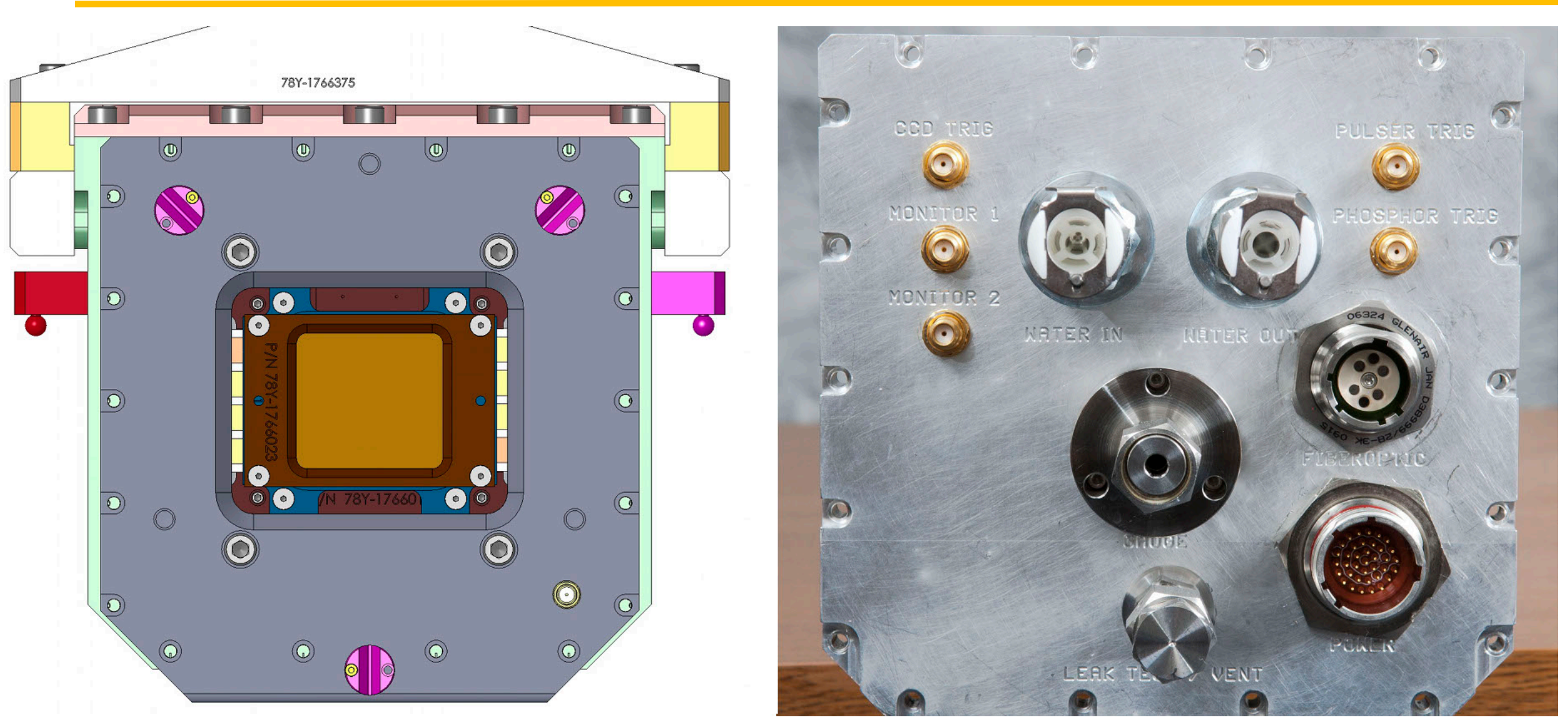

$\int_{\text {LATIONAL LABORATORY }}$

Slide 25 


\section{EMI Shielding, Strain Relief, SSD Location, LLNL PS}
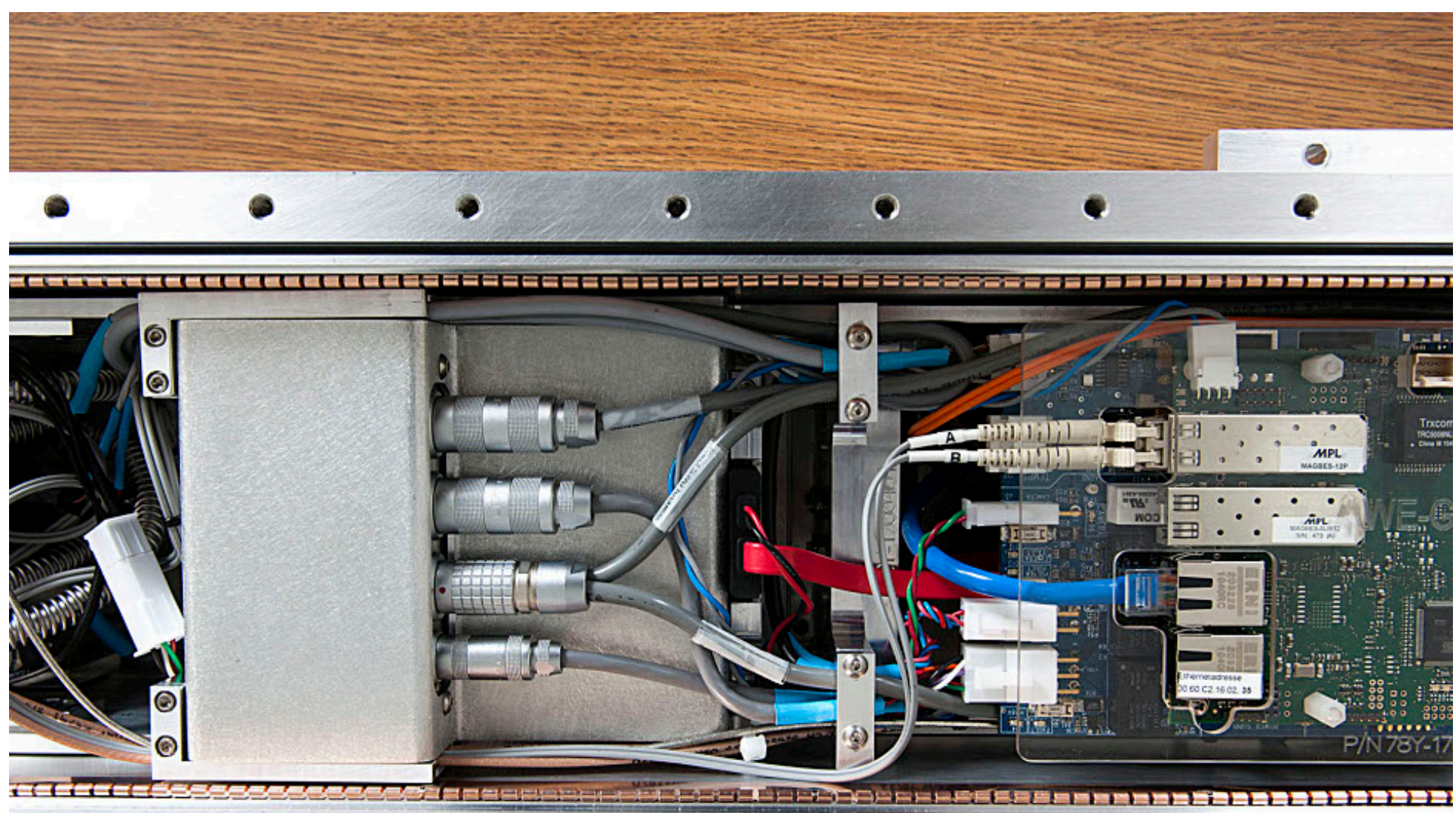

Ef

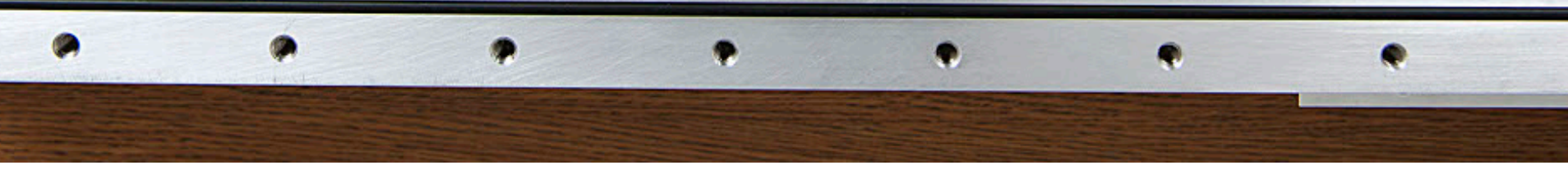

\section{- Los Alamos}




\section{Example Trident Test Data}




\section{Timing Shot GXD \#3 (Trident Shot 23121)}

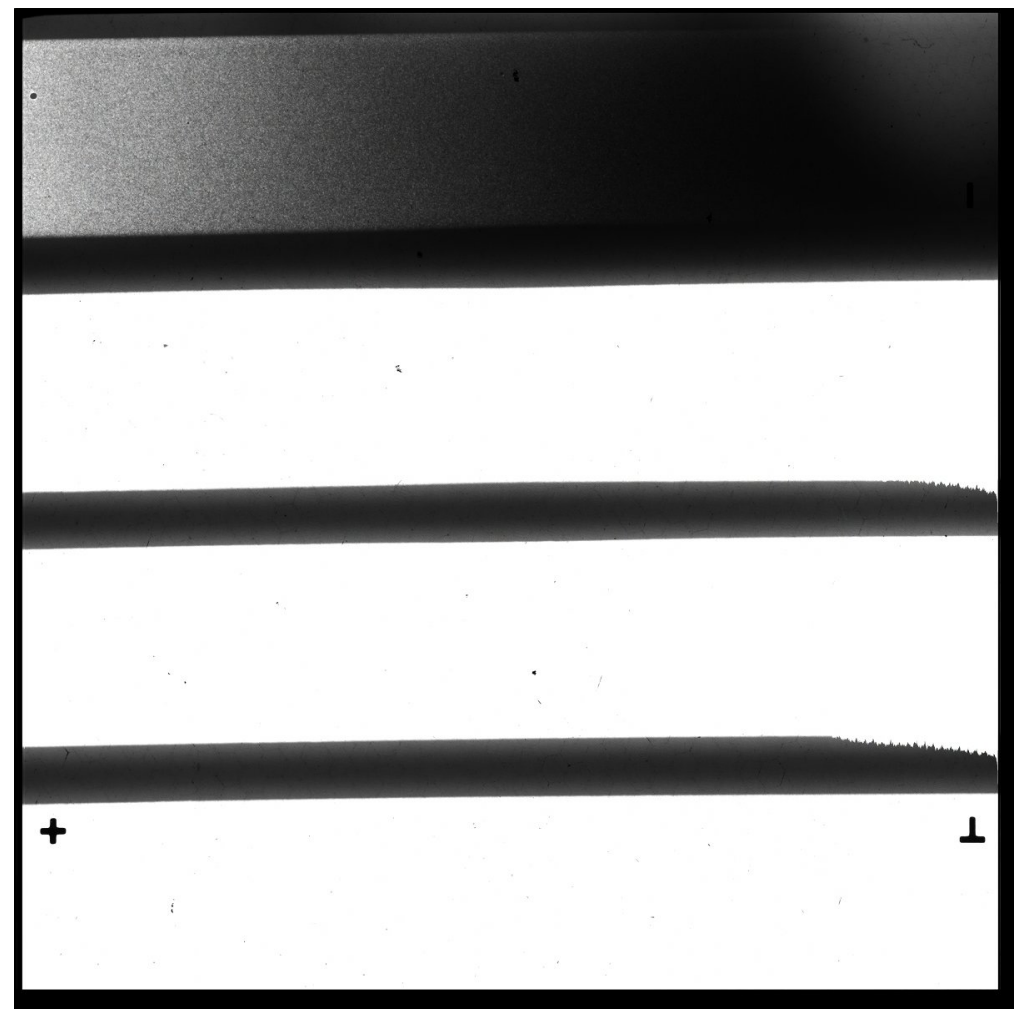




\section{Associated Timing Data Shot 23121}

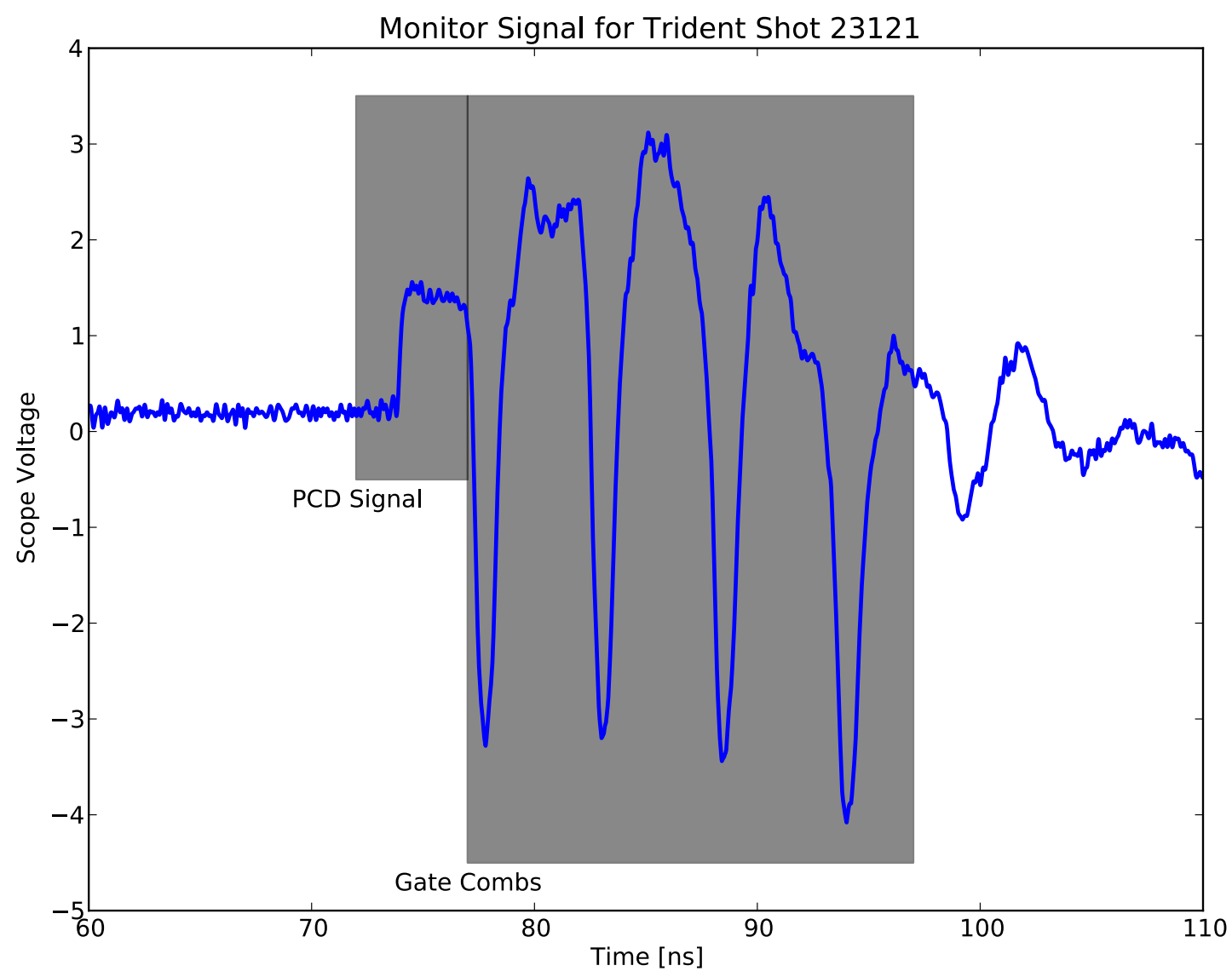




\section{GXD \#1 Trident Shot 23132}

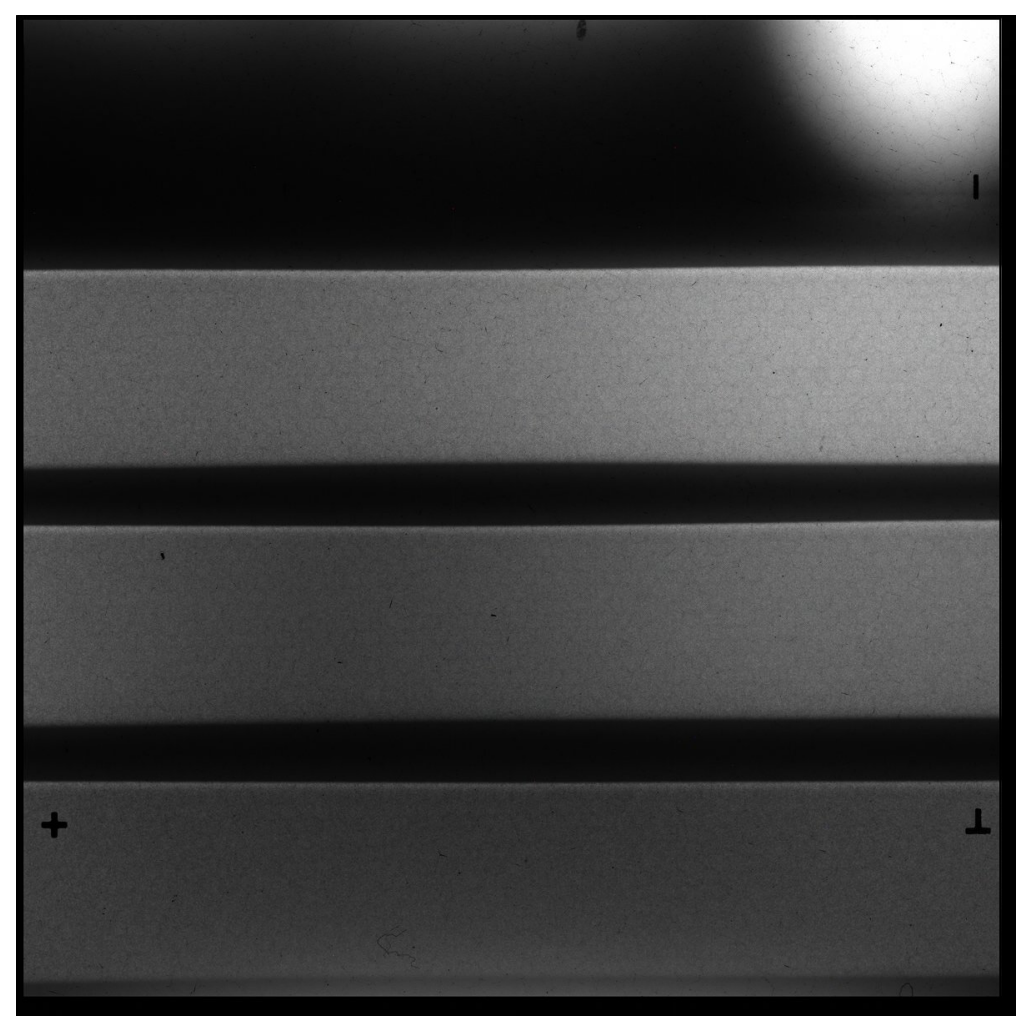




\section{Acknowledgements}

- Randy Johnson and the Trident Laser Team

- Kevin Oades and Simon Daykin and the Orion Laser Team

- Chris Bently and the AWE Manson Source Team

- Technical Photographer Joseph Cowan 